BLS 33, No 1 2007. DOI: http://dx.doi.org/10.3765/bls.v33i1.3523

(published by the Berkeley Linguistics Society and the Linguistic Society of America)

\title{
Towards a Substantively Biased Theory of Learning
}

\author{
SARA FINLEY and WILLIAM BADECKER \\ Johns Hopkins University
}

\section{Introduction}

There has recently been much debate concerning the nature of representations of phonological objects, particularly with regard to the role of abstract phonological constructs such as features and natural classes. This debate has extended to include differing notions about how phonological knowledge is learned and represented in the mind. The goal of this paper is to provide evidence for the psychological reality of abstract representations, including distinctive features. We argue for a theory of substantively biased learning in which learners are biased towards phonological patterns that are abstract, general, and correlate to cross-linguistic tendencies.

We review how the poverty of the stimulus paradigm for artificial grammar learning can be used to reveal that knowledge of vowel feature dependencies (height and tenseness) can lead to substantive biases about the level of generality in rule formation. This paper provides further data that substantive biases are abstract, and derived from constraints on the inventory of phonological segments.

This paper proceeds as follows. Section 1 provides background on the nature of vowel harmony and the type of substantive biases that are likely to form in vowel harmony systems. Section 2 discusses substantive biases in greater detail, including how it is possible to tap into the adult learner's biases. Section 3 describes the (Artificial Grammar) Poverty of the STIMulus PARAdigm, and the results of an experiment on height harmony. Section 4 presents and discusses the results of an experiment testing for generalization to novel suffixes in vowel harmony. Section 5 concludes with prospects for future experiments.

\section{Vowel Harmony}

Vowel harmony is a process whereby adjacent vowels are compelled to share the same value of a particular feature. There are several types of vowel harmony, each involving different features: height (Menomini (Cole and Trigo 1988)), back, round (Turkish (Kaun 2004)), tense/ATR (Lango (Archangeli and Pulleyblank 1994, Smolensky 2006)). Most harmony systems involve a single feature, however, there are some systems that involve multiple features, where one feature is 


\section{Sara Finley and William Badecker}

dependent on the presence of some other feature. For example, round harmony in Turkish applies only to high vowels. In Yalwelmani, round harmony applies only if the vowels already agree in height. In Menomini, height harmony is conditioned by tenseness; only tense vowels undergo harmony (Cole and Trigo 1988). This type of feature dependency in harmony is referred to as parasitic harmony, and it is not arbitrary. The features that can be parasitic on one another can only be features that are dependent on some dimension. For example, height and rounding are phonetically correlated such that the higher the vowel, the more likely it is to be round. Height and ATR are also phonetically correlated; low vowels are preferably lax ([-ATR]), and high vowels are preferably tense ([+ATR]). Phonetic correlations of features lead to markedness effects (Archangeli and Pulleyblank 1994) that in turn create restrictions in vowel harmony. The correlation of height and ATR produces parasitic harmony. In some languages with both tense and lax vowels, height/raising harmony applies only to tense vowels. Further, features that share little phonetic dependence, such as height and backness, do not interact parasitically in harmony systems. In the next section we provide some detail as to how these facts can manifest themselves as substantive biases in the learner.

\section{Substantive Biases}

Constraints on the typology of vowel harmony raise the question of whether or not the substance that forms these constraints is psychologically real. Following Wilson (2006), we define substance as "the system of categories that figure into the mental representation of linguistic knowledge" (945). This includes abstract representations that are used to describe phonological processes such as distinctive features, natural classes, as well as the basic vocabulary for describing phonological units: the syllable, prosodic word, stress, consonant, and vowel. Substance also refers to the psychological instantiation of theoretical notions that may be specific to a particular phonological framework, such as constraints in Optimality Theory (Prince and Smolensky 1993/2004).

Substantive biases guide learners towards phonological processes that conform to the substantive knowledge of the learner, acting as a prior on learning; the

learner forms hypotheses about the training data based on knowledge of what phonological processes should look like. If language learners have knowledge of features and natural classes, they will be biased to posit rules that make use of these features and natural classes. Further, if learners have knowledge about what makes a proper grammatical process, then they should be biased towards learning natural phonological processes.

\subsection{Testing for Substantive Biases}

Substantive biases are but one way to explain linguistic universals. An alternative account is offered in Evolutionary Phonology (Blevins 2004). According to Evolutionary Phonology, there is no need to posit independent knowledge of the constraints that govern cross-linguistic typology. As languages are learned, ease of articulation and misperception guide the learner. Because languages evolve by 


\section{Towards a Substantively Biased Theory of Learning}

universal constraints on misperception, languages evolve in very similar ways, and what looks like universal grammatical knowledge is actually epiphenomenal. Since language can be described in terms of misperception at the learning stage, it is redundant to assume that abstract representations of language are encoded in the minds of language learners.

One fruitful source of evidence for substantive biases in learning is the artificial grammar learning paradigm. In this paradigm, participants are exposed to a single morphophonological process of a novel language. This paradigm is efficient because it can be used to test a variety of hypotheses involving learning biases. For example, Wilson (2006) tested for substantive biases by training participants on a language game involving velar palatalization (e.g., [ki] $\rightarrow$ [tSi]). Half of the participants were exposed to high vowels only ([i]), and the other half of the participants were exposed to mid vowels only ([e]). Cross-linguistically, velar palatalization participates in an implicational relationship: if mid vowels trigger palatalization, then high vowels must also trigger palatalization, but not vice versa. If language learners have knowledge of this universal implication, then exposure to mid vowels should lead to generalization to high vowels, but exposure to high vowels need not lead to generalization to mid vowels. This is precisely what happened in Wilson's experiments - learners generalized to high vowels but not mid vowels. This result provides evidence that learners have substantive knowledge of perceptual and articulatory constraints even if their native language does not provide them with specific knowledge of the phonological rule that the constraints govern.

The asymmetric generalization found in Wilson's experiments supports the hypothesis that learners are biased towards perceptually natural processes. The fact that learners appear to be biased towards learning natural rules does not entail the inability to learn unnatural rules. However, the naturalness bias does suggest that the more a rule conforms to substantive knowledge, the easier it will be to learn. Conversely, the more a rule deviates from substantive biases, the more difficult that process will be to learn. Partial evidence for this can be found in Pycha et al.'s (2003) experiments in which a vowel harmony alternation was taught to three sets of participants. One group learned a phonologically natural pattern: vowel harmony. A second group was exposed to a phonetically natural but phonologically less natural pattern of disharmony, and a third group was exposed to a completely arbitrary morphophonological pattern. Evidence of learning was found in the phonologically and phonetically natural conditions, but there was no evidence of learning in the arbitrary condition. This supports the substantively biased learning hypothesis because the pattern that least resembled a well-formed grammatical process was the process that learners had the most difficulty learning.

The results of experiments testing directly for naturalness effects point in favor of substantive biases; no experiment has shown a bias towards learning an unnatural pattern over learning a natural pattern. However, because of difficulties in interpreting null results, a more promising source of evidence for biases can be 
found in tests for generalization to novel forms (Wilson 2006). Testing for generalization allows the researcher to make inferences about what hypotheses the learner has made. If the substantively biased learning hypothesis is correct, generalization to novel forms should reflect that fact.

The poverty of the stimulus paradigm (Wilson 2006) tests for substantive biases by specifically impoverishing the training set, and then testing learners on a novel, more enriched data set. The paradigm has the same logic as artificial grammar learning experiments that explore natural versus unnatural patterns. The difference is that in the poverty of the stimulus paradigm, the training in each condition is equally natural, but the predictions about generalization to novel forms may be different. In one condition, substantive biases may predict generalization to novel forms; in the other condition, substantive biases may predict no generalization to novel forms.

While the term 'poverty of the stimulus' is typically made in reference to arguments for nativist learning mechanisms, the poverty of the stimulus method for testing for substantive biases is compatible with both nativist and non-nativist accounts of learning. These biases could be learned or reflect innate constraints; in no way does the substantively biased hypothesis entail that these biases are present from birth.

In the next section, we describe how the poverty of the stimulus paradigm can be used to test for substantive biases in vowel harmony, particularly for biases based on feature dependence.

\section{The Poverty of the Stimulus Paradigm and Height Harmony}

As mentioned in Section 2, feature dependence can lead to cross-linguistic typologies of parasitic vowel harmony. Height harmony can be parasitic on ATR because these features are phonetically correlated, but because height and backness are not phonetically correlated, height harmony systems are much less likely to be parasitic on backness. If language learners have knowledge of feature dependencies between height and ATR, then this should be reflected in their generalizations to novel segments in an artificial grammar learning setting.

Finley and Badecker (2007) tested exactly this question. They trained participants on a height harmony rule that was either conditioned by tense vowels or conditioned by front vowels. In the Lax Hold-Out condition, participants were trained using only tense vowels ([i, u, e, o], e.g., [pigu, pigumi], [bego, begome]). After training, these participants were given a forced choice test that compared Old Items (items that were exactly what were given in training (e.g., [begome]), New Stem Items (items that contained the same vowels heard at training, but were not heard in training (e.g., [godeme]), and New Vowel Items (items that contained vowels not heard in training (e.g., [gIdImi]). The novel vowel items contained lax vowels ([I, E]) that were not heard at training. If participants who were exposed only to tense vowels hypothesize a general height harmony rule, they should extend the pattern to these lax vowels. However, if participants make use of their knowledge that height and tenseness are dependent features, they may posit a 


\section{Towards a Substantively Biased Theory of Learning}

parasitic harmony rule in which only tense vowels undergo height harmony. If this is the case, they will not generalize to lax vowels. In the Back Hold-Out condition, participants were exposed to the same stem-suffix alternation as in the Lax Hold-Out condition. The difference is that these participants only heard front vowels, ([I, E, i, e]). If participants make use of their knowledge that phonological processes tend to be general, and that height and back are not co-dependent features, then learners should posit a general height harmony rule, and extend the harmony pattern to novel vowels $([\mathrm{u}, \mathrm{o}])$.

Results indicated exactly that; participants extended the harmony pattern to back vowels but not to lax vowels. This supports the substantively biased learning hypothesis because knowledge of feature dependencies allowed learners to make differentiating hypothesis about the specificity of the height harmony rule that they were exposed to. Interestingly, generalization to one set of vowels over another cannot be explained in terms of acoustic distance to exemplars. The back vowels $[\mathrm{u}, \mathrm{o}]$ that participants were able to generalize to are acoustically far from the training set vowels [i, e], whereas the lax vowels [I, E] that participants failed to generalize to are acoustically close to the training set vowels [i, e]. If generalization were based on acoustic distance, then the opposite pattern would be expected.

The interpretation of these results relies on the assumption that substantivelybiased learning is general. If learning were specific (e.g., segment-based), then one should expect no generalization to novel segments when features were not dependent. The next section provides further support for the assumption that learners form generalizations through substantive biases. We present data from a new experiment that further supports the hypothesis that learning is both general and abstract.

\section{Generalization to Novel Suffixes}

The substantively biased learning hypothesis states that learners have access to knowledge about features, as well as more abstract linguistic principles, such as the fact that phonological processes tend to be general, applying to a wide variety of morphemes. If this is an accurate characterization of the knowledge that is brought to learning, then learners in the poverty of the stimulus paradigm should be able to generalize beyond the initial phonological process they are exposed to. In the vowel harmony experiments of Finley and Badecker (2007), participants were exposed to stem and stem + suffix alternations, with just one suffix exemplar (e.g., in the height harmony experiment discussed above, participants were exposed to the alternation of [-mi]/[-me]). If learners are able to form abstract vowel harmony rules from a single suffix alternation, they should be able to generalize to novel suffixes. In the present experiment, participants are exposed to a round harmony rule with a single suffix alternation, and are then tested on their generalization to a suffix with a novel vowel (e.g., from $[-\mathrm{mi}] /[-\mathrm{mu}]$ to $[-$ ge $] /[-g o])$. 


\section{Sara Finley and William Badecker}

An alternative grammatical interpretation of the result that learners form hypotheses for phonological processes in line with typological predictions (Finley and Badecker 2007; Wilson 2006) is that learners have direct knowledge of typlogical frequencies (e.g., in the form of statistical probablilities for rankings of particular constraints in an optimality theoretic grammar). Because the factors that lead to typological distributions (articulatory ease, perceptibility, learnability, etc.) are so tightly linked to typological frequencies themselves, it is possible that learners actually encode the probabilities of relative typological frequencies.

In the present experiment, it is possible to differentiate between these two grammatical hypotheses. High vowels are probabilistically better targets for round harmony than mid vowels. If this typological information is directly encoded in substantive biases, then language learners should generalize to high vowel suffixes, but not to mid vowel suffixes. However, the typological generalization that high vowels are better targets for round harmony is based on the fact that languages are more likely to have high round vowels in their vowel inventory than mid round vowels (Kaun 2004). If biases are derived from substantive constraints, such as the segmental inventory of the language, then language learners should generalize to both mid and high vowel suffixes, as long as the inventory contains both high and mid round vowels. In this experiment, all participants are trained with stems that contain both mid and high round vowels. When participants are exposed to mid vowel suffixes for the first time, they should already know that mid round vowels are allowed in the inventory of the novel language, and should have no restriction on whether mid vowels participate.

If substantive biases are based on inventory constraints, then participants should generalize to both high and mid vowel suffixes. Alternatively, if biases are based on knowledge of typological frequencies, then we should expect learners to behave differently in the two target hold-out conditions. In particular, they should generalize to novel high vowel suffixes, but not to novel mid vowel suffixes. In this experiment, we test substantive biases towards high targets to round harmony. Participants are exposed to round harmony where both mid and high vowel stems trigger harmony to either a high vowel suffix or a mid vowel suffix. Participants

are then tested on their generalization to novel suffix vowels, either high or mid, depending on their training.

\subsection{Method}

\subsubsection{Participants}

All participants were adult native English speakers with no knowledge of a vowel harmony language and did not participate in previous harmony experiments. Sixty-one Johns Hopkins undergraduate students participated for extra course credit. Participants were randomly assigned to one of three training groups: a Control group containing mixed harmony stems, a High Vowel Suffix condition and Mid Vowel Suffix condition. Final analyses included 20 participants in each group. All participants were screened based on a perceptual (AXB) task. Those 


\section{Towards a Substantively Biased Theory of Learning}

participants scoring less than 75 percent on this task were removed from the study. This occurred for one participant.

\subsubsection{Design}

The experiment consisted of a training phase followed immediately by a forcedchoice test. All phases of the experiment were presented using PsyScope (Cohen et al. 1993). The training consisted of 24 pairs of stems and stem plus suffix items in the experimental conditions (High and Mid Suffix Hold-Out conditions). Examples are provided in Table 1 below. The Control condition (mixed harmony stems) had 48 stems (this was double the number of stems so that Control training could consist of stems in the Mid and Low Hold-Out conditions, as well as disharmonic stems). Participants heard each stem-suffix pair five times, in one of two randomized orders. Training was followed by a 36-item forced-choice test. One item was harmonic (e.g., [bigimi]), while the other was disharmonic (e.g., [bigimu]). Each test item was from one of three conditions: Old Stems, New Stems, or New Suffix. The Old Stems condition contained items that appeared in training. New Stems items did not appear in training, but were drawn from the same vowel and consonant inventory as the training items. New Suffix test items consisted of an old stem suffixed with both a vowel and a consonant that did not appear in the training suffix.

(1) Table 1: Stimulus Design and Examples

\begin{tabular}{|c|c|c|c|}
\hline & Training Suffix & Novel Suffix & Training Examples \\
\hline \multirow[t]{2}{*}{ Mid Hold-Out } & {$[-\mathrm{mi}] /[-\mathrm{mu}]$} & {$[$-ge $] /[-$ go $]$} & [bodo-bodomu] \\
\hline & {$[-\mathrm{gi}] /[-\mathrm{gu}]$} & {$[-\mathrm{me}] /[-\mathrm{mo}]$} & [bodo-bodogu] \\
\hline \multirow[t]{2}{*}{ High Hold Out } & {$[$-ge $] /[-$ go $]$} & {$[-\mathrm{mi}] /[-\mathrm{mu}]$} & [bodo-bodoge] \\
\hline & {$[-\mathrm{me}] /[-\mathrm{mo}]$} & {$[-\mathrm{gi}] /[-\mathrm{gu}]$} & [bodo-bodogu] \\
\hline \multirow[t]{2}{*}{ Control } & $\bar{X}$ & $\begin{array}{l}{[-\mathrm{gi}] /[-\mathrm{gu}],} \\
{[-\mathrm{me}] /[-\mathrm{mo}]}\end{array}$ & [bodo, bido] \\
\hline & $\mathrm{X}$ & $\begin{array}{l}{[-\mathrm{mi}] /[-\mathrm{mu}],} \\
{[-\mathrm{ge}] /[-\mathrm{go}]}\end{array}$ & [bodo, bido] \\
\hline
\end{tabular}

In the present experiment participants were randomly assigned to one of three conditions: Control, Mid Suffix Hold-Out and High Suffix Hold-Out. In the Control condition, participants were exposed to 24 harmonic and 24 disharmonic two-syllable stems. In the Mid and High Hold-Out conditions, all stems contained the same vowel (e.g., [budo], [pimi], etc.). This eliminated the potential confound that learners might generalize to novel suffixes in both hold-out conditions if stem vowels contain evidence of spreading to both mid and high vowels (e.g., [budo] has left to right spreading of high to mid and [bodu] has left to right spreading of mid to high). Participants in each hold-out condition were exposed to one training suffix, and were asked to generalize to one novel suffix. The novel 
suffix always contained a different vowel, and a different consonant than the training suffix. For example, if a participant was trained with a [-mi]/[-mu] alternation, they were tested on the $[$-ge $] /[$-go $]$ alternation, and vice versa. Participants trained on [-gi]/[-gu] were tested on [-me]/[-mo], and vice versa. The suffix consonant was varied to ensure that generalization to novel suffixes did not depend on similarity to the training suffix (i.e., had the same consonant). Training was counterbalanced such that half of the participants in each condition received a suffix vowel with the bilabial nasal $[\mathrm{m}]([-\mathrm{mi}] /[-\mathrm{mu}]$ or $[-\mathrm{me}] /[-\mathrm{mo}])$ and the other half were trained using suffix involving a velar stop $[\mathrm{g}]([-$-gi $] /[-\mathrm{gu}]$ or $[-$ ge $] /[-\mathrm{go}])$. Participants in the Control condition were also counterbalanced to receive test items including $[-\mathrm{me}] /[-\mathrm{mo}]$ and $[-\mathrm{gi}] /[-\mathrm{gu}]$, or test items using the suffixes [-mi]/[-mu] and [-ge][-go].

The experiment finished with an AXB perception task as in Finley and Badecker (2007). This task required participants to identify which of two singlesyllable words contained the same vowel as the medial vowel. For example, if participants heard [bi, gi, du], the correct answer would be that the first syllable [di] contains a vowel identical to the vowel in the second syllable [gi].

\subsubsection{Stimuli}

All stimuli were recorded in a sound proof booth at $22,000 \mathrm{kHz}$ by a male speaker of American English with basic phonetic training and no knowledge of the experimental design. The intensity for all stimuli was scaled to $70 \mathrm{~dB}$. Sound editing was done using Praat (Boersma and Weenkin 2005). All stimuli contained segments drawn from the consonant and vowel inventories: [p, b, t, d, k, g, m, n] and $[i, \mathrm{u}, \mathrm{e}, \mathrm{o}]$.

Suffixed stimuli were created by splicing a pseudo-suffixed form with a central vowel $\left(\left[{ }^{\prime}\right] \quad\left(\right.\right.$ stem $+\left[\mathrm{m}^{\prime}\right]$; e.g., [badam $\left.\left.\left.{ }^{\prime}\right]\right)\right)$ and a spliced portion of a suffixed form of a central stem $[-\mathrm{mi}] /[-\mathrm{mu}]$, [-gi][-gu], [-me]/[-mo] or [-ge]/[-go]. For example, the form [dekemi] was created by crossing the stem portion of [dekem'] with the suffix portion of [ $\mathrm{d}^{\prime} \mathrm{k}^{\prime} \mathbf{m i}$ ]. This assured that the stimuli in the test condition, which contained both harmonic and disharmonic forms, had identical stem portions, and differed only by the suffix. This ensured that selection of the suffixed form was due to the suffix itself and not to an idiosyncrasy in the stem. The F1 and F2 values for each stem vowel were measured to ensure that proper the acoustic correlates were present.

Two different consonantal skeletons were made for each vowel pair for a total of 24 training words. Consonant skeletons were constructed so that each of the eight consonants $([\mathrm{p}, \mathrm{b}, \mathrm{t}, \mathrm{d}, \mathrm{k}, \mathrm{g}, \mathrm{m}, \mathrm{n}])$ occurred in word initial position three times and word-medial position three times. Vowel pairs were assigned to consonant skeletons randomly with the condition that any word too closely resembling an English word was avoided. Consonant skeletons were created in the same manner as the training for New Stems and New Suffix test conditions. Examples of stimuli used in the experiment are given in Table 1, above. 


\subsubsection{Procedure}

All participants were given written and verbal instructions. They were told that they would be listening to a language they had never heard before, and that they would later be asked about the language, but they need not try to memorize any forms they heard. Participants heard all stimuli over headphones. When training was complete, a new set of instructions appeared on the monitor. Participants were told that they would hear two words, one of which was from that language they just heard, and their task was to identify which word belonged to the language. Participants were told to respond as quickly and accurately as possible, and to make their responses after hearing both items. Participants were given a debriefing statement upon completion of the experiment (which took approximately 15 minutes). Although we did not probe participants as to whether they noticed the purpose of the experiment or obtained explicit knowledge of the phonological process, pilot subjects reported that they remained unaware of the purpose of the experiment, and had no explicit knowledge of the phonological rule. Participants were given a short break before beginning the AXB task after testing.

\subsection{Results}

Percent of harmonic responses were recorded for each subject for each of the training conditions. The mean responses for each test condition are presented in Figure 1, below.

(2) Figure 1: Percent of Harmonic Responses by Hold-Out Condition

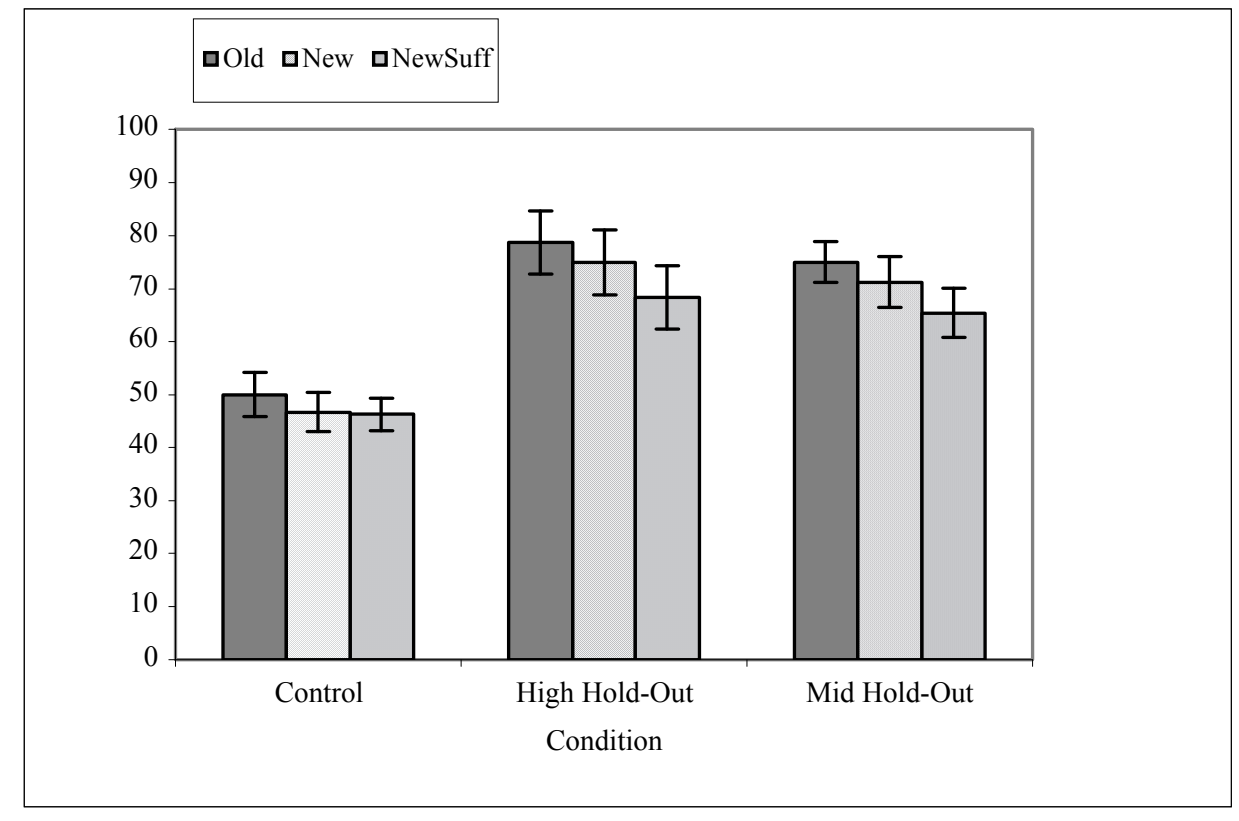




\section{Sara Finley and William Badecker}

Participants in each of the training conditions were compared to participants in the Control condition via a separate mixed design two-factor analysis of variance (ANOVA) with alpha set at $p=0.05$. The between-subjects factor was Training, with two levels in each ANOVA: the Control group and each Hold-Out condition. Test Items (Old Stems, New Stems, New Suffix) was a within-subjects factor nested under the between-subjects factor Training. All conditions involved between-item comparisons. 95\% confidence intervals (CI) are presented for each condition (Masson and Loftus 2003).

For the ANOVA comparing Controls to the High Hold-Out condition, there was a significant effect of Training $(\mathrm{F}(1,38)=652.70 ; \mathrm{p}<0.001)$, in that participants in the High Hold-Out condition (mean $=70.56, \mathrm{CI}= \pm 11.80$ ) were more likely to choose the harmonic option than participants in the Control condition $($ mean $=47.63, \mathrm{CI}= \pm 4.63)$. There was no effect of Test Item $(\mathrm{F}(2,38)=2.41 ; \mathrm{p}$ $>0.05)$, and no interaction between these factors $(F(2,76)=1.36 ; p>0.05)$.

For the ANOVA comparing Controls to Mid Hold-Out, there was a significant effect of Training $(\mathrm{F}(1,38)=19.01 ; \mathrm{p}<0.001)$; participants in the Mid Hold-Out condition (mean $=74.03, \mathrm{CI}= \pm 8.50$ ) were more likely to choose the harmonic candidate than participants in the Control condition. There was no effect of Test Item $(\mathrm{F}(2,38)=2.53 ; \mathrm{p}>0.05)$ and no interaction $(\mathrm{F}<1)$.

For the ANOVA comparing High Hold-Out to Mid Hold-Out, there were no effects for Training $(\mathrm{F}<1)$, and no interaction $(\mathrm{F}<1)$. There was an effect of Test Item $(\mathrm{F}(2,38)=8.45 ; \mathrm{p}<0.001)$.

To assess generalization to novel suffixes, a t-test was performed comparing the New Suffix condition of the Control condition to the New Suffix condition for each training condition. There was a significant difference between New Suffix test items and Controls for the High Hold-Out condition $(t=3.46 ; p<0.01)$ as well as the Mid Hold-Out condition $(\mathrm{t}=3.31 ; \mathrm{p}<0.001)$ in that participants were more likely to select the harmonic choice for New Suffix test items compared to Controls.

\subsection{Discussion}

Participants appear to have learned a general, robust round harmony rule, and were able to extend this rule to novel suffixes. These results provide evidence that learners form abstract, general hypotheses about the phonological processes that they are exposed to.

Participants generalized to both mid vowel and high vowel suffixes, indicating that learning is sensitive to inventory constraints, rather than a more direct encoding of typological frequencies (e.g., listings of probabilities of particular constraint rankings). This result leaves open the possibility that at least some portion of substantive biases are acquired rather than innately specified (and vice versa). The finding that language learners are not always sensitive to cross-linguistic frequencies of patterns has important implications for phonological theory. In particular, they suggest that a psychologically real theory of phonology need not directly encode typological frequencies in the grammar (e.g., in the form of 


\section{Towards a Substantively Biased Theory of Learning}

probability of specific constraint rankings). Rather, these frequencies can be derived from other substantive facts such as inventory constraints and perceptibility.

\section{Conclusion}

The results of the experiment presented in this paper, along with the results of previous experiments (Finley and Badecker 2007; Wilson 2006) support a substantively biased theory of learning. In this theory, learners are biased to form rules that are abstract, general and conform to typological generalizations. Learners are sensitive to inventory constraints, phonetic and phonological markedness as well as feature dependencies. They are also biased towards forming abstract rules, which are general and rely on feature-based representations.

What exactly is contained in substantive biases is still largely undiscovered. More research is needed to determine the precise nature of grammatical knowledge. Our future research will further investigate how typological predictions made by Optimality Theory (Prince and Smolensky 1993/2004) for vowel harmony manifest themselves in artificial grammar learning. Such issues include dominance and directionality in vowel harmony.

\section{Acknowledgments}

This research would not be possible without the helpful comments, questions and advice of the following: Paul Smolensky, Rebecca Morley, Becky Piorkowsky, Jenny Culbertson, Deepti Ramadoss, David Taylor, Iris Berent, Colin Wilson, Ariel Goldberg, Luigi Burzio, Donca Steriade, Charles Pickens, audiences at BLS 33, and the JHU Psycholinguistics Lab.

\section{References}

Archangeli, Diana, and Pulleyblank, Douglas. 1994. Grounded Phonology. Cambridge, MA: The MIT Press.

Beckman, Jill. 1997. Positional Faithfulness, Positional Neutralisation and Shona Vowel Harmony. Phonology 14:1-46.

Blevins, Juliette. 2004. Evolutionary Phonology: The Emergence of Sound Patterns. Cambridge: Cambridge University Press.

Boersma, Paul, and Weenink. 2005. Praat: Doing phonetics by computer.

Cohen, Jonathan, MacWhinney, Brian, Flatt, Matthew, and Provost, Jefferson.1993. PsyScope: A New Graphic Interactive Environment for Designing Psychology Experiments. Behavioral Research Methods, Instruments and Computers. 25:257-271.

Cole, Jennifer, and Trigo, Loren. 1988. Parasitic Harmony. In N. Smith and H. van der Hulst, eds., Features, Segmental Structures and Harmony Processes, 19-38. Dordrecht: Foris.

Finley, Sara, and Badecker, William. 2007. Artificial Language Learning 
and Feature-Based Generalization. Ms, Johns Hopkins University.

Kaun, Abigail. 2004. They Typology of Rounding Harmony. In B. Hayes and D. Steriade eds., Phonetics in Phonology, 87-116. Cambridge: Cambridge University Press.

Masson, Michael E, and Loftus, Geoffrey R. 2003. Using Confidence Intervals for Graphically Based Data Interpretation. Canadian Journal of Experimental Psychology 57:203-220.

Prince, Alan, and Smolensky, Paul. 1993/2004. Optimality Theory: Constraint Interaction in Generative Grammar. Cambridge: Blackwell.

Pycha, Anne, Nowak, Pawel, Shin, Eurie, and Shosted, Ryan. 2003. Phonological Rule Learning and its Implications for a Theory of Vowel Harmony. WCCFL 22:101-113.

Smolensky, Paul. 2006. Optimality in Phonology II: Harmonic Completeness, Local Constraint Conjunction, and Feature-Domain Markedness. In P. Smolensky and G. Legendre eds., The Harmonic Mind: From Neural Computation to Optimality-Theoretic Grammar, Volume II, 453-535. Cambridge, MA: The MIT Press.

Wilson, Colin. 2006. Learning Phonology with Substantive Bias: An Experimental and Computational Study of Velar Palatalization. Cognitive Science 30:945-982.

Sara Finley

Johns Hopkins University

Department of Cognitive Science

3400 N. Charles St.

Baltimore, MD 21818

finley@cogsci.jhu.edu

William Badecker

Johns Hopkins University

Department of Cognitive Science

3400 N. Charles St.

Baltimore, MD 21818

badecker@jhu.edu 